Journal of Engineering and Applied Sciences 14 (Special Issue 9): 10589-10593, 2019

ISSN: 1816-949X

(C) Medwell Journals, 2019

\title{
An Investigation of Improving EMG Control of Upper Limb Prostheses with Myo Armband Device for Transradial Amputees
}

\author{
${ }^{1}$ Mohammed S. Kadhim, ${ }^{1}$ Sadiq J. Abbas and ${ }^{2}$ Ali Hussain \\ ${ }^{1}$ Department of Biomedical Engineering, College of Engineering, \\ Al-Nahrain University, Baghdad, Iraq \\ ${ }^{2}$ Department of Biomedical Engineering, College of Engineering, \\ Baghdad University, Baghdad, Iraq
}

\begin{abstract}
The study improve sEMG signals, especially, for the transradial amputees, it investigated the classification accuracy of surface Electromyography (sEMG) with low-cost Myo armband device. Seventeen specific viable gestures used in daily-life were compared between two groups, the first one was represented by six transradial amputees and the second one was represented by six able-bodied subjects. In addition, the measured signals from forearm muscles were utilized to drive 3D printed prosthetic hand controlled by Pattern Recognition (PR) technique. In order to analyze the acquired sEMG signals, the PR system employed which consists of three main parts: segmentation, feature extraction and classification. The LDA classifier was employed in order to obtain optimum accuracy of the system. The classification accuracy of Myo armband showed high performance where the achieved classification accuracy for abled bodies subjects was more than (94.85\%) with LDA classifier and for transradial amputees subjects (88.2\%) with LDA classifier. Also, it was acceptable for 3D printed hand controller for 7 movements in study case of transradial amputees. The results may conclude that low cost Myo armband can be applicable for controlling 3D printed prosthetic hand to distinguish hand movements.
\end{abstract}

Key words: Wearable Myo armband, sEMG, Matlab, pattern recognition, LDA classifiers, 3D printed prosthetic hand

\section{INTRODUCTION}

Electromyogram (EMG) stem from three terms: electro which means pertaining to electric activity; Myo, which has a Greek root meaning muscle and gram which stands for recording. Electromyography or EMG, refers to recording of muscles electric activities. The upper limb prosthesis controlled by electromyography is refer to Myo electric control that is non-invasively recording via. surface electrodes built inside the socket of the upper limb prosthetic (like prosthetic for transradial amputees) (Smith et al., 2009). Transradial or below elbow amputation is partial removal of the forearm below the elbow joint which affects the hand function by hand loss (amputation) (Morgan et al., 2016). Hand loss which is an effective tool, leads to severe psychological and physical drawbacks (Cipriani et al., 2011). There are several solutions for this amputation type but still faces many challenges, like high cost and functionality limitation. One of this solution is Bionic 3 hand is it has multi-articulating fingers with a separated motor that actuate each finger with the thumb rotating manually and the hand is able to perform varying grip patterns with high price range about 30000 US\$ (Anonymous, 2009). Other solutions were came with the developments of electronics technology and embedded systems, from this solution is Myo armband was used for controlling the prosthetic hand where the work was done on healthy people (normal subjects) (Donovan et al., 2016). Myo armband is a wearable myoelectric systems, low cost, embedded processing and wireless interfacing in an independent portable device, the Myo armband device (Thalmic Labs) is commercially available (Luh et al., 2016). Pattern Recognition (PR) technique has been used for multi-functional transradial prosthesis control, it was offered intuitive control and multiple movements controlling (Scheme and Englehart, 2011). In this study, Myo armband was used for controlling the low cost 3D printed prosthetic hand with pattern recognition technique which it means the data can be easy analyzed and the price reduction can gets. Pattern recognition technique is divided into segmentation, feature extraction and classification. sEMG signals can be classified by using several classifying algorithms, such as Linear Discriminant Analysis (LDA) (which is used in this paper), Support Vector Machines (SVM), Artificial Neural Networks (ANN) and K-Nearest Neighbor (KNN) (Mendez et al., 2017). The range of amplitude of the sEMG signal from $0-10 \mathrm{mV}$ (from peak to peak) or 0-1.5 $\mathrm{mV}$ (rms) and the applicable frequency (energy) 
range of EMG signal is from $0-500 \mathrm{~Hz}$ with the dominant frequency (energy) range from $50-150 \mathrm{~Hz}$ in the EMG signal application (De Luca, 2002). This encouraged the use of the Myo armband despite its design at a low sample rate about $200 \mathrm{~Hz}$ (Luh et al., 2016). When a pattern has been specified, a prosthesis controller is commanded to improve the movement $(\mathrm{Li}, 2011)$. This study aims to aid the progress in robotic 3D printed hand prosthetics, make a statistical and scientific database of electromyography, develop and test algorithms for 13 movements for transradial amputees subjects with pattern recognition technique.

\section{MATERIALS AND METHODS}

The proposed system, shown in Fig. 1 by using the sEMG signals from Iraqi subject with the use of low cost Myo armband device.
Firstly, we record the sEMG signal, secondly then split it into $25 \%$ testing set and $75 \%$ training set (1 trial for testing \& 3 trial for training). sEMG is considered as noisy and non-stationary, it can be considered as stationary if short time intervals (segments) for this signal was used. The segment length can be defined as the length of the time-slot of sEMG signal which used to implement feature extraction. The length of the segment and segmentation scheme are the main significant points that need to be found. Generally, two types of segmentation schemes can be used: the first is disjointed scheme, the second is overlapped scheme which was used in this study (Englehart and Hudgins, 2003). After that, the features were extracted finally the classifier have been train and its performance was estimated by calculating confusion matrix and Majority Vote (MV) and total accuracy all that done by 'mec-main' matlab codes. Materials used for recording sEMG in this work are shown in Fig. 2.
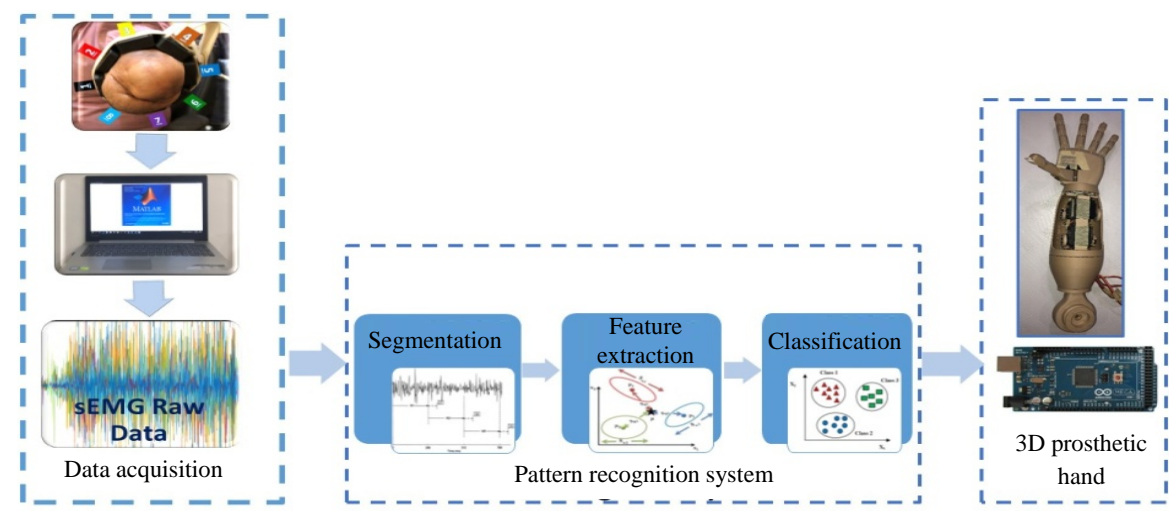

Fig. 1: Pattern recognition control system

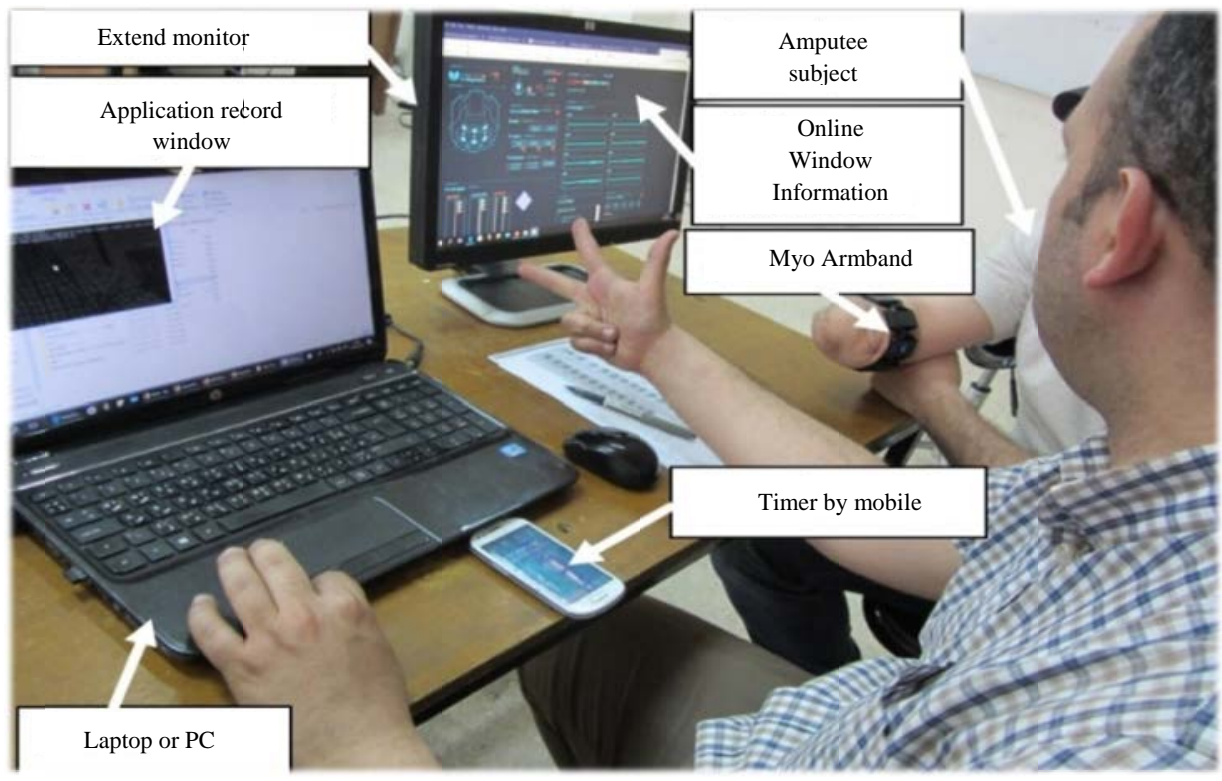

Fig. 2: Materials of experimental work used in this study 
The Myo armband device is shown in Fig. 3. It has eight stainless channel for pick sEMG and all the references of the 8 channel were connected with a professional and deliberate manner to reduce the noise (Luh et al., 2016).

Lenovo Laptop was used for easy recording, due to more mobility to record data. The Lenovo laptop has the following specifications, Intel core i7-7500, 7th Gen with CPU @2.70 GHz(4CPUs), random access memory (RAM) $8.192 \mathrm{GHz}$ and 4.262 GB display card (VGA) has name Intel "NVIDIA GEFORCE" was used in this study.

Matlab R2018b. (student license) with price from the official website 87 US\$ was used in this study which took

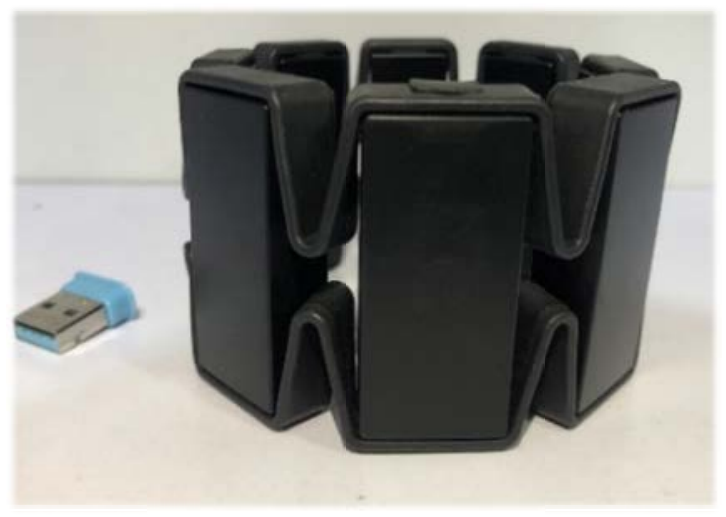

Fig. 3: Myo Armband with Bluetooth stick several hours to download from the company's website after purchase. Arduino MEGA 2560 and Arduino Software Integrated Development Environment (IED) which is an open source was also used in this study.

Low Cost 3D Printed Hand with German cosmetic cover for upper limb Fig. 4 was used, due to it is cheap price, open source, suitable for different age groups, suitable for difficult economic situation for most people, the selection of the "Humanoid Torso Proto 1" robotic hand (Arthur, 2018) was done. The humanoid proto 1 robotic hand is very light, powerful and elegant type of prosthetic hand.

Methods of the analysis of data of twelve subjects were selected in this study and they were divided into two groups: The first one was used for transradial amputee which included the study of 6 transradial amputee subjects as shown in Table 1.

And the second one is used for normal subjects which included the study of 6 normal subjects as shown in Table 2.

The basic setup and relax sitting position is very important, the level of cooperation, attention and responsiveness of the person is also important. The way of guidance of the person who is amputated or normal by instruction from the researcher or by the presentation of pictures or video, shows the movements to be carried out, the duration of movement and duration of rest and the number of repeat, each gesture lasted 6 second and repeated four times, it must be mentioned that the contraction power of the target muscles was moderate.

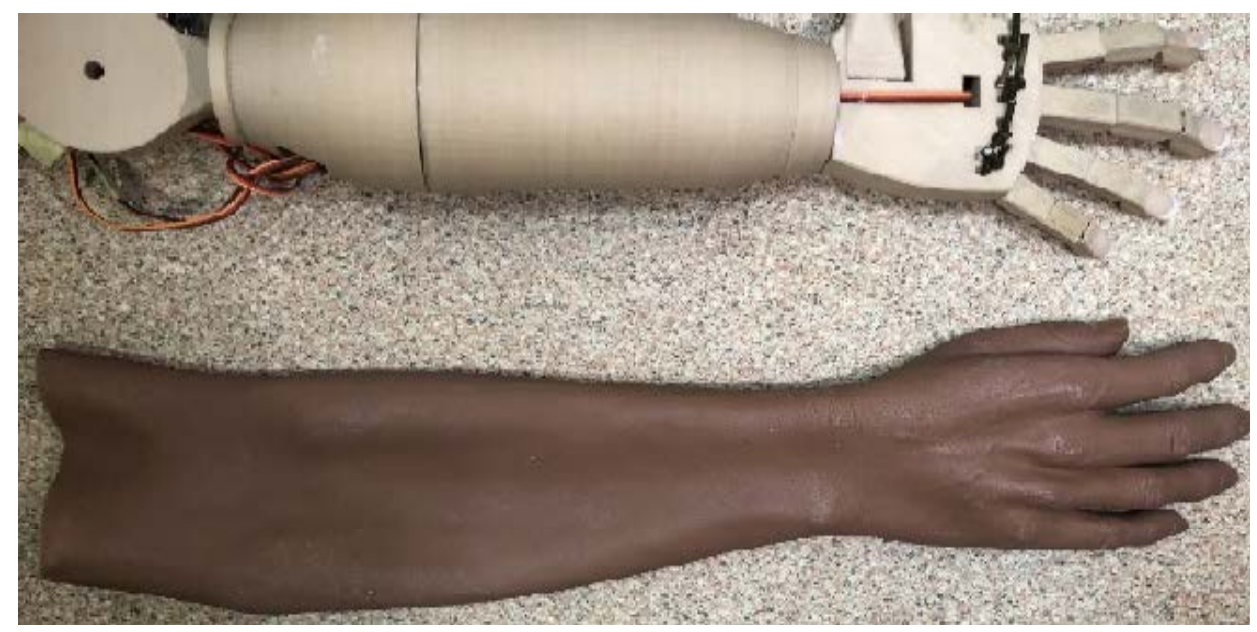

Fig. 4: 3D printed hand with German cosmetic cover for upper limb

Table 1: Database of 6 Iraqi transradial amputee subjects

\begin{tabular}{|c|c|c|c|c|c|c|c|}
\hline Amputee subject & Age/Years & Gender & Height $(\mathrm{cm})$ & Wight (kg) & Dominant hand & Tested amputee arm & Amputation period/Years \\
\hline Amp 1 & 60 & Male & 168 & 69 & Right & Right & 36 \\
\hline Amp 2 & 52 & Male & 178 & 77 & Right & Left & 1 \\
\hline Amp 3 & 43 & Male & 170 & 72 & Left & Left & 27 \\
\hline Amp 4 & 41 & Male & 178 & 84 & Left & Left & 15 \\
\hline Amp 5 & 23 & Male & 171 & 61 & Right & Right & 1 \\
\hline Amp 6 & 22 & Male & 176 & 65 & Right & Right & 14 \\
\hline
\end{tabular}


J. Eng. Applied Sci., 14 (Special Issue 9): 10589-10593, 2019

Table 2: Database of 6 Iraqi able-bodied (Normal) subjects

\begin{tabular}{lclccll}
\hline Normal subject & Age/Years & Gender & Height $(\mathrm{cm})$ & Weight $(\mathrm{kg})$ & Dominant hand & Tested arm \\
\hline Nor 1 & 67 & Female & 170 & 90 & Left & Left \\
Nor 2 & 35 & Male & 177 & 70 & Right & Right \\
Nor 3 & 32 & Male & 177 & 85 & Right & Right \\
Nor 4 & 31 & Male & 176 & 68 & Right & Right \\
Nor 5 & 27 & Male & 183 & 95 & Right & Right \\
Nor 6 & 15 & Male & 167 & 60 & Right & Right \\
\hline
\end{tabular}

The mean and the standard deviation were calculated for most results and a comparison was made between the two groups. The 17 gestures were recorded from each subject. The recording data from 6 healthy subjects and 6 transradial amputees were saved in database. There was a difficulty to collect database required to complete this work, due to the obstacles for accessing to cooperating subjects, all that because of lack an organized database in health sites in Iraq. The collected data were analyzed with PR in MATLAB program. The steps are pre-processing technique, data segmentation, feature extraction and classification. It also investigates the best robust movement's control of 3D Humanoid prosthetic hand. Because sEMG signals is complicated in nature it needs for filtration for noise removing but Myo armband device dose all that and no extra filters are needed to work.

\section{RESULTS AND DISCUSSION}

This study included the results of analytical software operations of sEMG signals where features have been extracted and classified with construction of an integrated PR Software system. Myo armband device was used for data acquisition for 12 subjects (six transradial amputees and six normal subjects) with 17 movements per each subject (four poor performance movements were removed from each transradial amputees to obtain more accurate results). One classifier type LDA was used. The system was based on Matlab program which registered with the original version of MathWork and it is upgradeable. All the required codes and information were saved on the MATLAB Software and thus, computer was used to complete this work. Control of this artificial 3D hand with online Myo armband device will discuses and the possibility of manufacturing a complete intelligent artificial hand which can be traded to low-income Iraqi amputees.

System affected by the length of the sampling window: The results of the classifier and computational speed were significantly affected by the length of the segmentationsampling window in pre-processing step of pattern recognition. More than one length of the window was used in overlapped segmentation scheme. The best results were obtained when used of length 41 samples (around $200 \mathrm{msec}$ ) and showed the highest results for all 12 persons. For reliable results, this value must be fixed for all patients and readings.

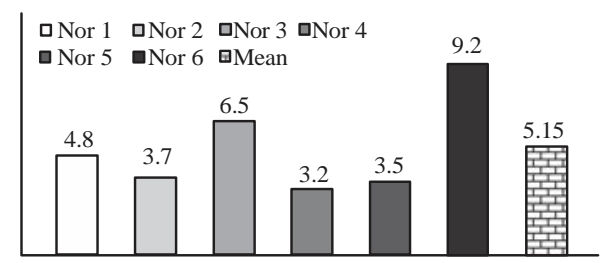

Fig. 5: Classification error rates for 17 movement for all 6 normal subjects (All 8 channels of Myo armband)

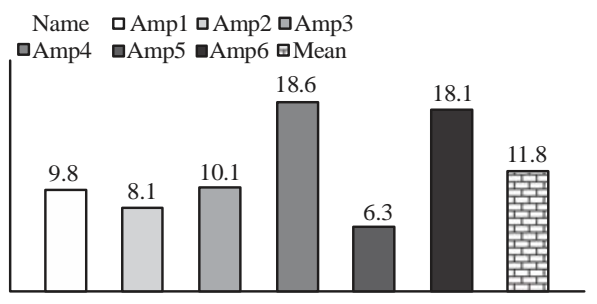

Fig. 6: Classification accuracy and error rates (\%) for 13 movements for all 6 amputees subjects (All 8 channel of Myo armband)

System affected by feature type: The choice of number and type of feature is very important but is considered better than the selection of the classifier themselves. Six types of time domain features were used. The best features results came with Root Mean Square (RMS), Waveform Length (WL), Zero Crossings (ZC), Slope Sign Changes (SSC), Integral Absolute Value (IAV), and 4 orders Auto Regression Features (AR). For reliable results, this type of feature must be fixed for all patients and readings.

Results for normal Iraqi subjects: In Fig. 5, classification error rates for 17 movement for all 6 normal subjects presented that is collected by Myo armband which is moderate and acceptable as comparing with high end sEMG devices results. Normal subjects were represented as (Nor 1, Nor 2, Nor 3, Nor 4, Nor 5, Nor 6).

Results for transradial amputees Iraqi subjects: The classification accuracy error rates (\%) of 13 gestures for 6 transradial amputee subjects were represented as (Amp 1, Amp 2, Amp 3, Amp 4, Amp 5, Amp 6) shown in Fig. 6 this results of classification accuracy is acceptable in spite of long amputation period. Related results and 
classification accuracy for 13 movements of transradial amputees subjects by used of Myo armband which it is very good for the performance of this device after analyzing the data collected.

\section{CONCLUSION}

To evaluate low-cost Myo armband and proving its efficiency in medical and research applications, the following can be concluded:

The classification accuracy of Myo armband showed best performance which achieved classification accuracy for able-boded subjects of more than (94.85\%) with LDA classifier and for transradial amputees subjects (88.2\%) with LDA classifier which acceptable for 3D printed hand controller for 13 movements.

The LDA classifier is accurate and applicable for PR technique to analyze sEMG signals. The Time Domain (TD) features are accurate and applicable for PR technique. The 3D printed hand is low cost manufactured and the grip strength is suitable for moderate use such as simple movements like gestures.

Finally, as a conclusion, the proposed PR system achieved promising results and has the capability to be marketed as complementary with high cost commercial devices and prosthesis hand in future and suitable for children because of the continuous change in their bodies as a result of growth.

\section{REFERENCES}

Anonymous, 2009. Touch bionics focuses on i-LIMB hand, upper body prosthetics. Robotics Business Review, USA. https://www.roboticsbusinessreview. com/health-medical/touch-bionics-focuses-on-i-lim b-hand-the-award-winning-bionic-hand/

Arthur, J.N., 2018. Water wheel with drink can water supply tank. My Mini Factory, London, UK. https://www.myminifactory.com/object/3d-print-w ater-wheel-with-drink-can-water-supply-tank-56472

Cipriani, C., M. Controzzi and M.C. Carrozza, 2011. The Smarthand transradial prosthesis. J. Neuroeng. Rehabil., 8: 1-13.

De Luca, C.J., 2002. Surface electromyography: Detection and recording. DelSys Incorporated, 10: 1-10.
Donovan, I., K. Valenzuela, A. Ortiz, S. Dusheyko and H. Jiang et al., 2016. MyoHMI: A low-cost and flexible platform for developing real-time human machine interface for myoelectric controlled applications. Proceedings of the 2016 IEEE International Conference on Systems, Man and Cybernetics (SMC), October 9-12, 2016, IEEE, Budapest, Hungary, ISBN: 978-1-5090-1898-7, pp: 004495-004500.

Englehart, K., and B. Hudgins, 2003. A robust, real-time control scheme for multifunction myoelectric control. Biomed. Eng. IEEE Trans., 50: 848-854.

Li, G., 2011. Electromyography Pattern-RecognitionBased Control of Powered Multifunctional Upper-Limb Prostheses. In: Advances in Applied Electromyography, Mizrahi, J. (Ed.). IntechOpen, London, UK., ISBN:978-953-307-382-8, pp: 1-21.

Luh, G.C., Y.H. Ma, C.J. Yen and H.A. Lin, 2016. Muscle-gesture robot hand control based on sEMG signals with wavelet transform features and neural network classifier. Proceedings of the 2016 International Conference on Machine Learning and Cybernetics (ICMLC) Vol. 2, July 10-13, 2016, IEEE, Jeju, South Korea, ISBN:978-1-5090-0391-4, pp: 627-632.

Mendez, I., B.W. Hansen, C.M. Grabow, E.J.L. Smedegaard and N.B. Skogberg et al., 2017. Evaluation of the Myo armband for the classification of hand motions. Proceedings of the 2017 International Conference on Rehabilitation Robotics (ICORR), July 17-20, 2017, IEEE, London, UK., ISBN:978-1-5386-2297-1, pp: 1211-1214.

Morgan, E.N., B. Kyle Potter, J.M. Souza, S.M. Tintle and G.P. Nanos, 2016. Targeted muscle reinnervation for transradial amputation: Description of operative technique. Tech. Hand Upper Extremity Surg., 20: 166-171.

Scheme, E. and K. Englehart, 2011. Electromyogram pattern recognition for control of powered upper-limb prostheses: State of the art and challenges for clinical use. J. Rehabil. Res. Dev., 48: 643-659.

Smith, A., P. Nanda and E.E. Brown, 2009. Development of a myoelectric control scheme based on a time delayed neural network. Proceedings of the 2009 Annual International Conference on the IEEE Engineering in Medicine and Biology Society, September 3-6, 2009, IEEE, Minneapolis, Minnesota, USA., pp: 3004-3007. 